\title{
A Method for Polarizing More Number of Impurity-Vacancy Dipoles
}

\author{
Jai Prakash 1,2*, Devendra Prasad ${ }^{3}$ \\ ${ }^{1}$ Department of Applied Sciences \& Humanities, Buddha Institute of Technology, Gorakhpur, India \\ ${ }^{2}$ Previously, Department of Physics, D.D.U. Gorakhpur University, Gorakhpur, India \\ ${ }^{3}$ Department of Physics (Basic Sciences), U P Textile Technology Institute, Kanpur, India \\ Email: *jpgkp.2010@rediffmail.com
}

Received 3 September 2015; accepted 26 February 2016; published 29 February 2016

Copyright (C) 2016 by authors and Scientific Research Publishing Inc.

This work is licensed under the Creative Commons Attribution International License (CC BY).

http://creativecommons.org/licenses/by/4.0/

cC) (7) Open Access

\begin{abstract}
Free rotating impurity-vacancy (IV) dipoles in an alkali halide matrix are polarized to the extent of $1 / 3$ of the total number of IV dipoles. An experimental procedure is suggested in this article which will help in the polarization of IV dipoles to the extent of $2 / 3$ of the total number of IV dipoles. In the suggested experimental procedure, the electric field will be applied at first in one direction and then will be applied in succession in opposite direction. Ionic thermocurrent technique is employed to ascertain the increase in polarization of IV dipoles.
\end{abstract}

\section{Keywords}

Ionic Thermocurrent, Frozen-In Polarization, Rapid Cooling Rate, Impurity-Vacancy Dipole

\section{Introduction}

Whenever a divalent impurity ion is introduced in an alkali halide matrix, a vacancy is created in its neighborhood for the sake of charge compensation. Vacancy created at the next nearest neighbour position with respect to the impurity ion forms an impurity-vacancy (IV) dipole. It would be justified to mention that at any instant of time, the vacancy will be located at one of the twelve equivalent sites around the impurity ion along $<110>$ direction. The dipole moment $(p)$ of the created IV dipole will be $\sqrt{2} e a$ where $e$ is the electronic charge and $a$ is the interatomic distance. In the absence of electric field, IV dipoles are randomly oriented in the system. In the presence of polarizing electric field $E_{P}$, these 12 equally probable orientations of IV dipoles will group into 3 categories as (a) 4 orientations with the energy $-p E_{P}$ favouring preferred direction of orientation, (b) 4 orientations with zero energy lying perpendicular to the electric field $E_{P}$ and (c) 4 orientations with the energy

*Corresponding author. 
$p E_{P}$ lie along unpreferred direction of orientation. For the IV dipoles of category (a) in the preferred orientation direction, the polarization $P$ after the application of electric field increases exponentially [1] [2] with the passage of time $t$ as

$$
P=P\left(T_{p}\right)\left[1-\exp \left(-\frac{t}{\tau}\right)\right]
$$

where $P\left(T_{p}\right)$ is the saturation polarization at the polarization temperature $T_{p}$ and $\tau$ is the relaxation time at the temperature corresponding to time $t . P\left(T_{p}\right)$ is expressed through

$$
P\left(T_{p}\right)=\frac{\alpha N_{d} p^{2} E_{P}}{k T_{p}}
$$

where $N_{d}$ is the number of IV dipoles per unit volume, $k$ is the Boltzmann's constant and $\alpha$ is a dimensionless geometrical parameter which depends on the possible orientations of the dipole in the crystal structure. For free rotating dipoles, $\alpha$ has a value of $1 / 3$. Thus, saturation polarization $P\left(T_{p}\right)$ is expressed as

$$
P\left(T_{p}\right)=\frac{N_{d} p^{2} E_{P}}{3 k T_{p}}
$$

After the application of electric field $E_{P}$, it is observed that $P$ increases exponentially with the passage of time $t$ in accordance with Equation (1) as shown in Figure 1. R.T in the figure represents the room temperature. It would be justified to mention that IV dipoles of category (b) with zero energy will not contribute to the polarization being perpendicular to $E_{P}$. Further, those IV dipoles wih energy $p E_{P}$ of category (c) will also not contribute to the polarization being in the unpreferred orientation position. Thus, it is only $1 / 3$ of the total number of IV dipoles in the specimen which will be polarized after the application of $E_{P}$ to the extent presented by Equation (3). Saturation polarization $P\left(T_{p}\right)$ is obtained through the application of electric field $E_{P}$ at the polarization temperature $T_{p}$ for a time large in comparison to its relaxation time at $T_{p}$ as shown in the range B to C of Figure 1. For recording the ionic thermocurrent (ITC) measurement [3] [4] such polarized IV dipoles with the electric field $E_{P}$ still on are rapidly cooled down to a fairly low temperature $T_{0}$ as shown in

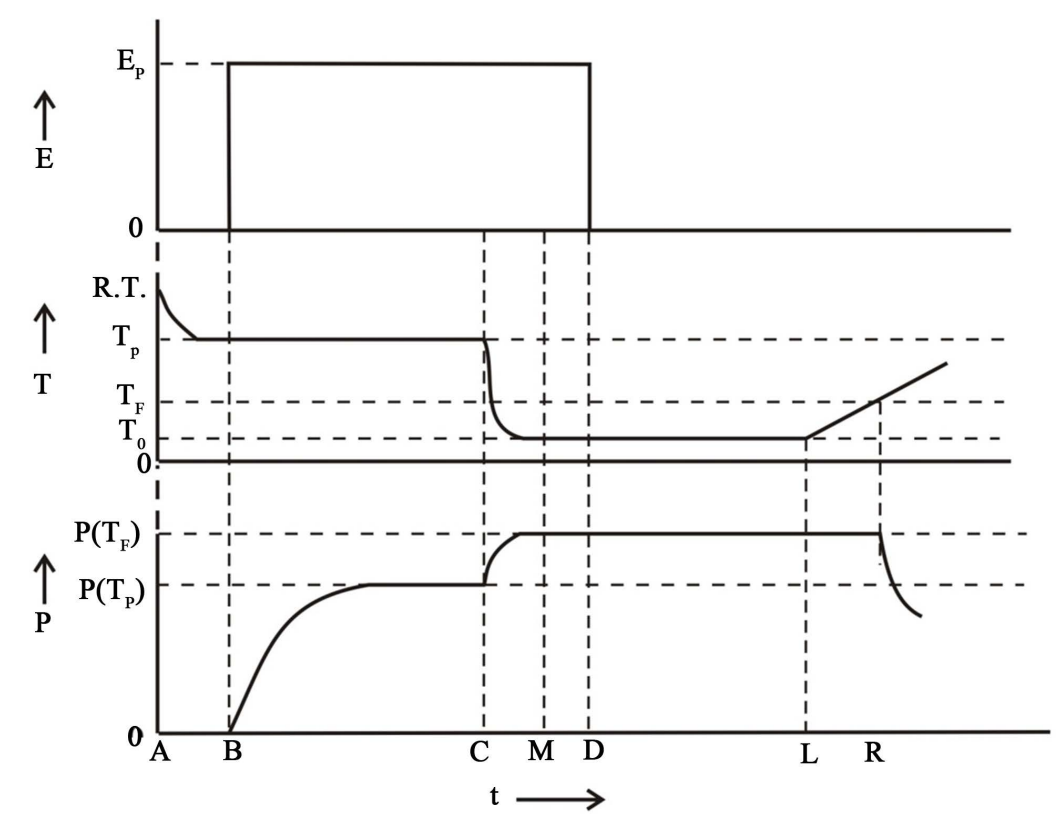

Figure 1. Polarization $(P)$ of the specimen as a function of time $(t)$. 
the range $\mathrm{C}$ to $\mathrm{M}$ of Figure 1. For $T_{p}$ close to room temperature, $P\left(T_{p}\right)$ is achieved quickly whereas at lower temperatures, more time is needed because of large relaxation time at these temperatures in accordance with the Arrhenius relation [5]

$$
\tau=\tau_{0} \exp \left(\frac{E_{a}}{k T}\right)
$$

where $\tau$ is the relaxation time at the temperature $T, \tau_{0}$ is the fundamental relaxation time or relaxation time at infinite temperature and $E_{a}$ is the activation energy for the orientation of IV dipole. The temperature $T_{0}$ is hence chosen suitably in such a way that the relaxation time at $T_{0}$ is quite large or practically infinite. Thus, motion of IV dipole is hindered at $T_{0}$. At such a low temperature the electric field is switched off at the point $\mathrm{D}$ of Figure 1, leaving the IV dipoles in the polarized condition since the relaxation time being practically infinite at $T_{0}$. Consequently, these polarized IV dipoles are frozen-in in the crystalline lattice. Similar experimental steps up to the point $\mathrm{D}$ are also followed in Figure 2, the details of which will be given elsewhere later on. The extent of frozen-in polarization is given by Equation (3). It has been an awaited requirement that the polarization in a system be increased to explore its technical potentialities. To have the maximum utilization and technical application of the dielectric properties of the system, workers engaged in the field of dielectric relaxation studies were putting lot of efforts to increase the polarization of the system. With this aim in view, an experimental method has been proposed in this article which helps in increasing the extent of polarization. Thus, frozen-in polarized IV dipoles of category (a) can be joined with those IV dipoles of category (c) following the experimental procedure suggested in this article leading to increment in the polarization.

\section{Suggested Experimental Technique}

After getting the frozen-in polarized IV dipoles at the point D of Figure 1, the specimen is kept for some time at $T_{0}$ in the range $\mathrm{D}$ to $\mathrm{L}$ for establishing equilibrium condition at $T_{0}$. The system is then heated at the point $\mathrm{L}$ of Figure 1 following a constant linear heating rate $b$ according to

$$
T=T_{0}+b t
$$

Consequently, the temperature $T$ increases from $T_{0}$ with the passage of time $t$ as shown in Figure 1. When the temperature is increased to $T_{F}$ corresponding to point $\mathrm{R}$ of Figure 1, the frozen-in polarized IV dipoles become able to depolarize or disorient [6] and continue depolarizing exponentially with the increasing temperature resulting in the appearance of depolarization current $I$. Resulting depolarization current $I$ can be recorded expe-

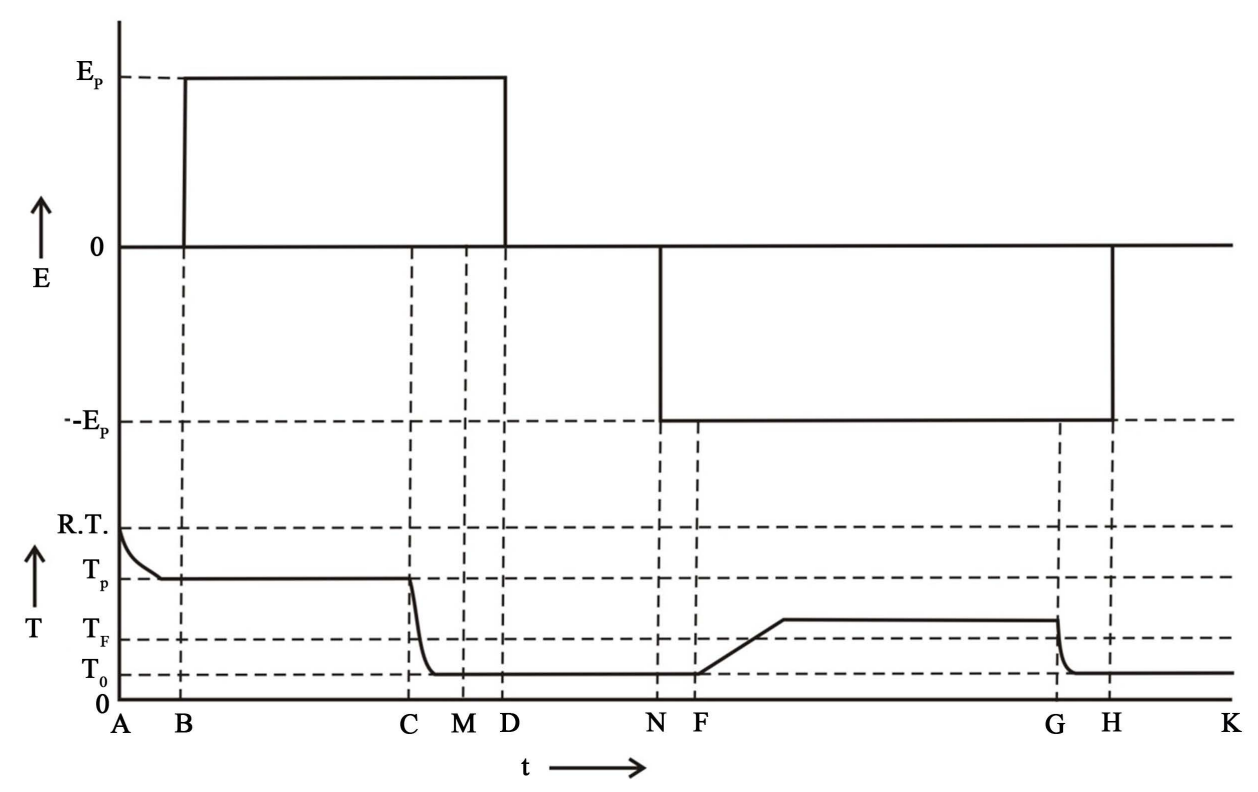

Figure 2. Schematic diagram of experimental steps of polarization in the two runs of ITC spectra. 
rimentally as a function of $T$. Plot of $I$ versus $T$ is known as an ITC spectrum [3] [4]. A representative ITC spectrum is shown in Figure 3 with the respective locations of $T_{0}, T_{F}$ and $T_{p}$. An ITC spectrum is an asymmetrical curve as obvious from Figure 3. It is very much similar to a thermoluminescence (TL) glow curve involving a monomolecular or first order kinetics. ITC spectrum is mathematically represented by [3] [4]

$$
I=\left(\frac{Q_{0}}{\tau_{0}}\right) \exp \left[\left(-\frac{E_{a}}{k T}\right)-\left(\frac{1}{b \tau_{0}}\right) \int_{T_{F}}^{T} \exp \left(-\frac{E_{a}}{k T^{\prime}}\right) \mathrm{d} T^{\prime}\right]
$$

where $Q_{o}$ is the total charge released during an ITC run and $T^{\prime}$ is an arbitrary temperature in the temperature range $T_{F}$ to $T$. The value of $Q_{o}$ is determined from the area of the ITC spectrum using the relation [3] [4]

$$
Q_{o}=\frac{1}{b} \int_{T_{F}}^{\infty} I \mathrm{~d} T
$$

where $\int_{T_{F}}^{\infty} I \mathrm{~d} T$ represents the total area of the ITC spectrum enclosed in the temperature range $T_{F}$ to $\infty$. It is obvious from Figure 3 that depolarization current does not start appearing at $T_{0}$ rather it starts appearing at a slightly higher temperature $T_{F}$ when frozen-in polarized IV dipoles become able to depolarize or disorient [6]. Peak value $I_{m}$ of ITC spectrum appears at the temperature $T_{m}$ as shown in the figure. The values of $T_{0}, T_{F}$, $T_{p}, I_{m}, T_{m}$ and $b$ are obtained experimentally. These values are needed for characterizing the ITC spectrum and for evaluating the relevant parameters like $E_{a}$ and $\tau_{0}$. It is obvious from Figures 1-3 that $T_{0}, T_{F}$ and $T_{p}$ are different such that $T_{p}>T_{F}>T_{0}$. Polarization temperature $T_{p}$ will lower down to $T_{F}$ while rapidly cooling down the specimen from $T_{p}$ to $T_{0}$. Consequently, the system will effectively be polarized corresponding to temperature $T_{F}$. Below $T_{F}$ the relaxation time becomes practically infinite and hence the system is not polarized up to $T_{0}$. Equation (3) for $T_{F}$ will, hence, be represented by

$$
P\left(T_{F}\right)=\frac{N_{d} p^{2} E_{P}}{3 k T_{F}}
$$

where $P\left(T_{F}\right)$ is the equilibrium polarization established at $T_{F}$. The value of $T_{F}$ in Equation (8) depends on the rate of rapid cooling [7] [8]. During rapidly cooling down the specimen from $T_{p}$ to $T_{0}$ in the presence of $E_{P}$, the polarization increases from $P\left(T_{p}\right)$ to $P\left(T_{F}\right)$ exponentially as shown in Figure 1. The extent of increase in $P\left(T_{p}\right)$ depends on the rate of rapid cooling. Higher rate of rapid cooling results in less increment in

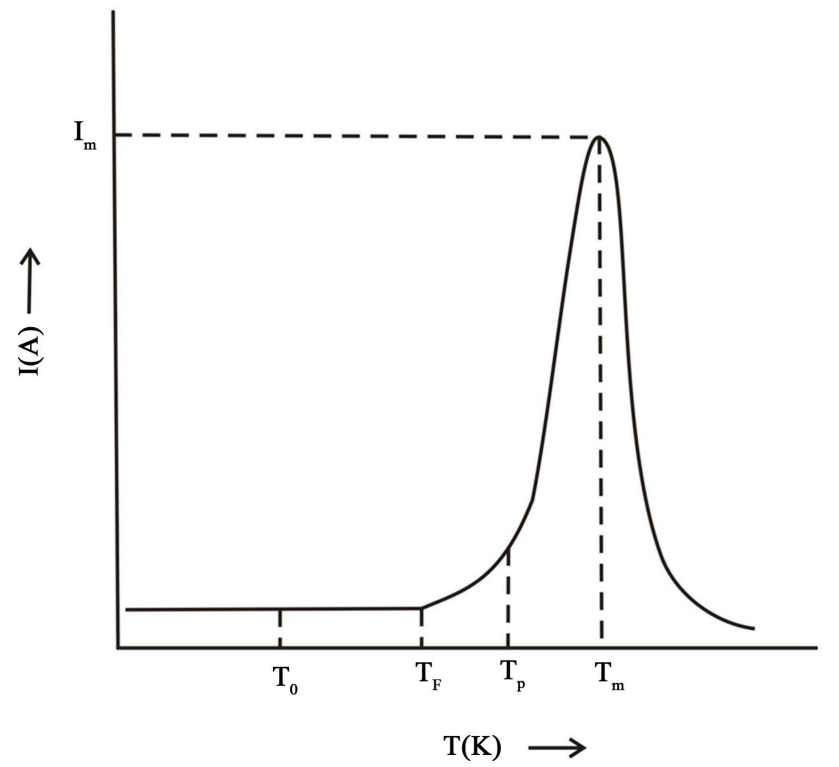

Figure 3. A representative ITC spectrum where $I$ is recorded as a function of $T$. 
polarization because lowering down of the temperature from $T_{p}$ to $T_{F}$ becomes less and less with the increasing rate of rapid cooling [7] [8]. If the rate of rapid cooling is infinitely large, the increment in the polarization will be zero and will remain equal to $P\left(T_{p}\right)$ because of the fact that time available for polarization of specimen is much less than the corresponding relaxation time at that temperature. Since, $T_{F}$ depends on the rate of rapid cooling and hence $P\left(T_{F}\right)$ also depends consequently on the rate of rapid cooling. It has been established by Christodoulides [9] that in ITC measurement where the specimen is rapidly cooled down to a fairly low temperature, the effective polarization temperature is less than $T_{p}$. This effective polarization temperature is appearing as $T_{F}$ in this article. Thus, $T_{0}<T_{F}<T_{p}$ finds a justification and happens to be in accordance with the observations of Christodoulides. It must be mentioned, however, that in various references reported in the literature, $P\left(T_{p}\right)$ has invariably been inadvertently used [1] [2] in place of $P\left(T_{F}\right)$.

Corresponding to recorded ITC spectrum expressed through Equation (6), depolarization current density $(J)$ is represented by

$$
J=\frac{I}{S}=\frac{Q_{0}}{S \tau_{0}} \exp \left[-\frac{E_{a}}{k T}-\frac{1}{b \tau_{0}} \int_{T_{F}}^{T} \exp \left(-\frac{E_{a}}{k T^{\prime}}\right) \mathrm{d} T^{\prime}\right]
$$

where $S$ is the area of the crystal specimen. Obviously $J$ is depolarization current per unit area. The quantity $\left(Q_{o} / S\right)$ in Equation (9) represents the frozen-in polarization $P\left(T_{F}\right)$ at the temperature $T_{F}$ as [3] [4]

$$
P\left(T_{F}\right)=\frac{Q_{0}}{S}
$$

Combination of Equations (8) and (10) gives

$$
Q_{o}=\frac{N_{d} p^{2} E_{p} S}{3 k T_{F}}
$$

Obviously, $Q_{o}$ depends on the area of the crystal specimen under investigation.

To get the number of polarized IV dipoles increased, an experimental procedure is proposed in this article. Frozen-in polarized IV dipoles are obtained following the experimental steps up to point D of Figure 1. ITC spectrum corresponding to frozen-in polarized IV dipoles is recorded experimentally following a constant linear heating rate. Such a plot is shown in Figure 3. Now the experimental procedure is repeated on the same specimen and in similar experimental conditions up to point $\mathrm{D}$ of Figure 2. The specimen will now be maintained at $T_{0}$ in the range $\mathrm{D}$ to $\mathrm{N}$ of Figure 2, to have the equilibrium condition established at $T_{0}$. At the point $\mathrm{N}$, the polarizing electric field $E_{P}$ will be applied in the opposite direction with reversed polarity. At the point $\mathrm{F}$ of Figure 2, the specimen will be slowly heated to a temperature slightly higher than $T_{F}$ in the presence of reversed applied electric field and maintained at this temperature for a time large in comparison to its relaxation time at this temperature. Consequently, those IV dipoles of category (c) which were initially in the unpreferred orientation position will now become automatically in the preferred orientation position in accordance with the reversed applied electric field direction and hence will remain oriented as such. Whereas already frozen-in polarized IV dipoles of category (a) will flip their orientation position in accordance with the direction of the reversed applied electric field. Thus, IV dipoles of categories (a) and (c) will become oriented along the same direction. IV dipoles of category (b) will remain unaffected being perpendicular to the reversed applied electric field $E_{p}$. At the point $G$ of Figure 2, the specimen will be rapidly cooled down to $T_{0}$ keeping the same rate of rapid cooling as at the point $C$ of both the Figure 1 and Figure 2. The reversed applied electric field will be switched off at the point $\mathrm{H}$ of Figure 2. Thus, the polarized IV dipoles of catagories (a) and (c) are obtained in the frozen-in condition in the crystalline lattice. The resulting frozen-in polarization after following the suggested experimental procedure will obviously be due to IV dipoles of catagories (a) and (c). Thus, the total number of frozen-in polarized IV dipoles will be doubled i.e. $2 / 3^{\text {rd }}$ of the total number of IV dipoles. Obviously, one will thus get more number of frozen-in polarized IV dipoles through the suggested experimental procedure. Expression for net frozen-in polarization due to IV dipoles of catagories (a) and (c) will hence be given by

$$
\left[P\left(T_{F}\right)\right]_{n e t}=\frac{2 N_{d} p^{2} E_{P}}{3 k T_{F}}
$$


The specimen will now be kept at the temperature $T_{0}$ in the range $\mathrm{H}$ to $\mathrm{K}$ of Figure 2, to establish equilibrium of net frozen - in polarized IV dipoles. ITC spectrum recorded after executing the suggested experimental procedure up to point $\mathrm{K}$ will show the peak value almost 2 times more in comparison to previously recorded ITC spectrum provided the constant linear heating rate $b$ and other experimental conditions are maintained in both the experimental runs of ITC. It will be advantageous to reiterate just for the sake of clarification that $1^{\text {st }}$ ITC spectrum of Figure 4 is recorded after following the experimental steps up to point D of Figure 1 whereas $2^{\text {nd }}$ ITC spectrum is recorded after following the experimental steps up to point $\mathrm{K}$ of Figure 2. Further, electric field $E_{P}$ in one direction in the range $\mathrm{B}$ to $\mathrm{D}$ will be applied for less time than that in the opposite direction with reversed polarity in the range $\mathrm{N}$ to $\mathrm{H}$ because relaxation time at a temperature slightly higher than $T_{F}$ is much more in comparison to the relaxation time at $T_{p}$. The peak of the ITC spectrum in both the runs will be located at the same position of temperature $T_{m}$ since $b$ is kept same [10] [11]. The values of $E_{a}$ and $\tau_{0}$ shall remain the same in both these cases being characteristic features of the specimen under investigation. A little difference may appear in the two recorded ITC spectra particularly because of the fact that $T_{F}$ used in above equations depends on the rate of rapid cooling [7] [8] which may differ in the two runs of ITC. It is expected that a fairly good agreement will be obtained between experimental and theoretical values. Thus the polarization of the system can be increased following the suggested experimental technique.

\section{Results and Discussion}

ITC spectrum recorded corresponding to the polarization conditions up to point D of Figure 1 following a constant linear heating rate will look like curve 1 of Figure 4. If we record another ITC spectrum on the same specimen in similar experimental conditions following the polarization procedure up to point $\mathrm{K}$ of Figure 2 and heating the specimen with the same constant linear heating rate, the recorded spectrum will appear like curve 2 of Figure 4. It is obvious from the figure that the peak of the ITC spectrum is doubled while rest of the parameters remains the same. A slight difference in the peak values of ITC spectra in the cases 1 and 2 of Figure 4 from the theoretically anticipated value may be due to slight difference in the values of rate of rapid cooling in the two cases. It is a known fact that $T_{F}$ depends on the rate of rapid cooling. Consequently, the values of $T_{F}$ while rapidly cooling down the specimen may be slightly different in the two cases of Figure 4. However, in the record of ITC spectra of Figure 4, the values of $T_{F}$ are observed to be the same because ITC spectra are recorded after slowly heating the specimen following same constant linear heating rate. Depolarization current starts appearing at $T_{F}$ in both the runs of ITC spectra while slowly heating the specimen following a constant linear heating rate. It is because of this reason that $T_{F}$ established while rapidly cooling down the specimen

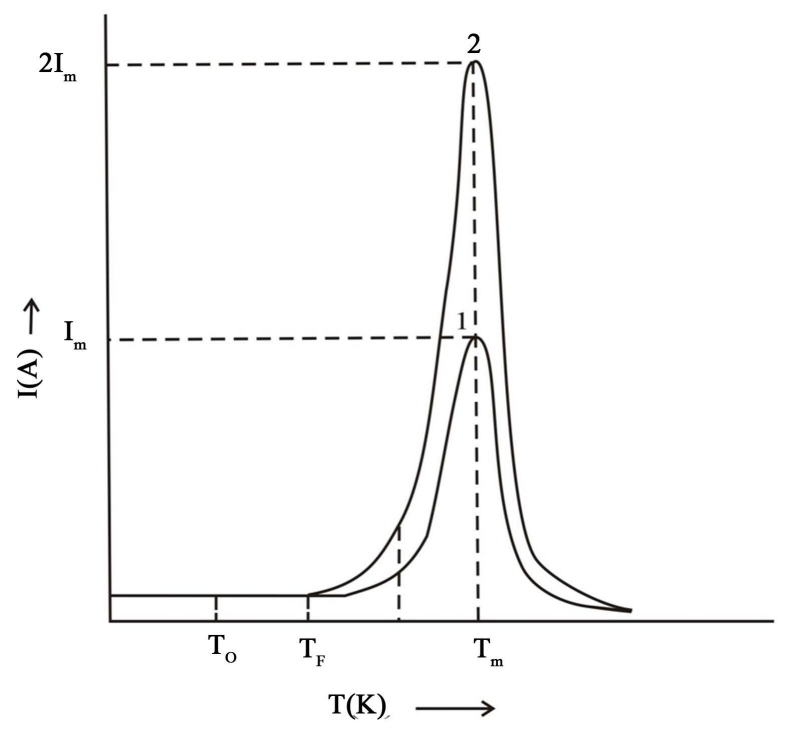

Figure 4. ITC spectra recorded following same constant linear heating rate and in similar experimental conditions on the same specimen: 1 . Polarized following the experimental process up to point D of Figure 1. 2. Polarized following the experimental process up to point K of Figure 2. 
may be different than that recorded during ITC spectra. Higher value of $T_{F}$ gives less value of $P\left(T_{F}\right)$ resulting in less value of $I_{m}$ and vice-vresa. Higher value of $T_{F}$ due to changes in the experimental conditions may be the probable reason for peak value in case 2 of Figure 4 being $<2 I_{m}$. If the value of $T_{F}$ while rapidly cooling down the specimen in both the runs of ITC spectra happens to be more in $1^{\text {st }}$ run than that of $2^{\text {nd }}$ run due to different rates of rapid cooling in the two cases, one would observe the peak value of ITC spectrum in $2^{\text {nd }}$ run $>$ $2 I_{m}$. It is obvious from Equations (2) and (3) that the polarization $P$ is inversely proportional to $T$. If $T_{F}$ is less, $P\left(T_{F}\right)$ will obviously be more resulting in the more value of $Q_{o}$ through Equation (10). Higher value of $Q_{o}$ leads to more value of $I_{m}$ according to [1] [2]

$$
I_{m}=\frac{Q_{0}}{\tau_{0}} \exp \left[-\frac{E_{a}}{k T_{m}}-\frac{1}{b \tau_{0}} \int_{T_{F}}^{T_{m}} \exp \left(-\frac{E_{a}}{k T^{\prime}}\right) \mathrm{d} T^{\prime}\right]
$$

It has to be taken care of in Equation (13) that $T=T_{m}$ and integration is performed in the range $T_{F}$ to $T_{m}$. Thus, the peak value of ITC spectrum in the $2^{\text {nd }}$ run after following different experimental steps up to point $K$ of Figure 2 may be different than $2 I_{m}$. It would be justified to mention that it is experimentally very difficult to maintain the same rate of rapid cooling in the two cases being a very fast changing process. However, constant linear heating rate can be maintained easily while recording the ITC runs resulting in the location of ITC peak at the same temperature $T_{m}$. In this reference it would be advantageous to go through the experimental observations of thermally stimulated polarization current (TSPC) measurements by Kristianpoller and Krish [12] recorded on $\mathrm{BaF}_{2}$ crystals doped with $\mathrm{Tb}^{3+}$. While recording the TSPC spectra on this system they have kept (i) $E_{p}=0$, (ii) keeping $E_{p}$ at some suitable value for the purposes of polarization and (iii) keeping $E_{p}$ same as in case (ii) but applied in the opposite direction while rapidly cooling down the specimen. Subsequently, they have observed different TSPC peaks in these three referred cases. In case (iii), the TSPC peak has experimentally been found to be 2 times more [12] in comparison to that obtained in case (ii). Thus, theoretically expected results proposed in this article, get a justification and a positive support through these experimental findings.

Increment in the number of polarized IV dipoles is also obtained during photo-induced polarization (PIP) when the specimen is simultaneously polarized and irradiated in its suitable absorption band [13] [14]. Increment to the extent of $70 \%$ has been observed during PIP [15]. It must be noticed that the increase in polarization during PIP takes place via electronic excited state [15] [16]. In the present suggested experimental procedure the polarization is increased through the orientation of IV dipoles taking place in the electronic ground state following bistable model [17]. Thus, the number of IV dipoles to the extent of $2 / 3^{\text {rd }}$ of the total number of IV dipoles can be polarized through the suggested experimental procedure proposed in this article.

\section{Acknowledgements}

The authors are thankful to Dr. R.A Agrawal, the chairman of the institute, for providing necessary facilities. They are also thankful to Mr. A. K. Srivastava, Mrs. Vandana Gupta and Mr. A.K. Arya for their help in computer graphics.

\section{References}

[1] Vanderschueren, J. and Gasiot, J. (1979) Topics in Applied Physics. In: Bräunlich, P., Ed., Thermally Stimulated Relaxation in Solids, Vol. 37, Springer-Verlag, Berlin.

[2] Chen, R. and Kirsh, Y. (1981) Analysis of Thermally Stimulated Processes. Pergamon Press, New York.

[3] Bucci, C., Fieschi, R. and Guidi, G. (1966) Ionic Thermocurrents in Dielectrics. Physical Review Letters, $148,816$. http://dx.doi.org/10.1103/PhysRev.148.816

[4] Prakash, J. (1990) Reorientational Behavior of Impurity-Vacancy Dipoles—Part I. Indian Journal of Pure and Applied Physics, 28, 365.

[5] Arrhenius, S. (1889) Zeitschrift für Physikalische Chemie, 4, 226.

[6] Prakash, J., Nishad, A.K. and Rahul (1984) Dielectric Relaxation Time of Frozen-in Impurity-Vacancy Dipoles in Potassium Chloride. Japanese Journal of Applied Physics, 23, 1404. http://dx.doi.org/10.1143/JJAP.23.1404

[7] Harasta, V. (1969) Fyz. Casop (Czech.), 19, 232.

[8] Prakash, J. Thermoluminescence Glow Curve Involving Any Extent of Retrapping or Any Order of Kinetics. Pramana-Journal of Physics, under Publication. 
[9] Christodoulides, C. (1988) Effective Temperature of Polarization in Measurements of Thermally Stimulated Depolarization Currents. Physica Status Solidi, a109, 611.

[10] Prakash, J., Rahul and Nishad, A.K. (1985) Heating Rate Dependence of Peak and Position of Maximum Depolarization Current in ITC Measurements. Physica Status Solidi, a91, 685.

[11] Kunze, I. and Müller, P. (1969) Relaxation von Fehlstellendipolen in AgCl-Kristallen. Physica Status Solidi, 33, 91102. http://dx.doi.org/10.1002/pssb.19690330106

[12] Kristianpoller, N. and Krish, Y. (1979) Thermally Stimulated Polarization Currents in Barium Fluoride. Journal of Physics: Solid State Physics, C12, 1079.

[13] Prakash, J. and Fischer, F. (1976) Optically Stimulated Polarization of S--- -Vacancy Dipoles in KI. Journal de Physique (France), 37, c7-c167.

[14] Prakash, J. (1990) Reorientational Behavior of Impurity-Vacancy Dipoles-Part II. Indian Journal of Pure and Applied Physics, 28, 667.

[15] Prakash, J. and Fischer, F. (1977) Ionic Thermocurrent and Optical Absorption Studies of S--_-Vacancy Dipoles in KI. Physica Status Solidi, a39, 499.

[16] van Turnhout, J. (1980) Topics in Applied Physics. In: Sessler, G.M., Ed., Thermally Stimulated Discharge of Electrets, Vol. 33, Springer-Verlag, Berlin.

[17] Nowick, A.S. (1972) Point Defects in Solids. In: Crawford Jr., J.H. and Slifkin, L.M., Eds., Plenum Press, New York. 